\title{
Site-Selective Orbital Interactions in an Ultrathin Iron-Carbene Photosensitizer Film
}

\author{
Robert H. Temperton, Nils W. Rosemann, Meiyuan Guo, Niclas Johansson, Lisa A. Fredin, Om Prakash,
} Kenneth Wärnmark, Karsten Handrup, Jens Uhlig, * Joachim Schnadt,* and Petter Persson*

Cite This: J. Phys. Chem. A 2020, 124, 1603-1609

Read Online

\section{ACCESS \\ Џll Metrics \& More \\ Article Recommendations \\ Supporting Information}

ABSTRACT: We present the first experimental study of the frontier orbitals in an ultrathin film of the novel hexa-carbene photosensitizer $\left[\mathrm{Fe}(\mathrm{btz})_{3}\right]^{3+}$, where btz is $3,3^{\prime}-$ dimethyl-1,1'-bis( $p$-tolyl)-4,4'-bis(1,2,3-triazol-5-ylidene). Resonant photoelectron spectroscopy (RPES) was used to probe the electronic structure of films where the molecular and oxidative integrities had been confirmed with optical and X-ray spectroscopies. In combination with density functional theory calculations, RPES measurements provided direct and site-selective information about localization and interactions of occupied and unoccupied molecular orbitals. Fe 2 p, N 1s, and C 1s measurements selectively probed the metal, carbene, and side-group contributions revealing strong metal-ligand orbital mixing

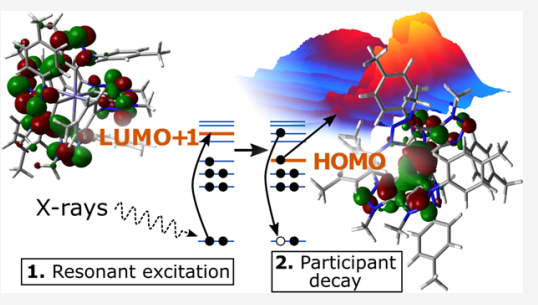
of the frontier orbitals. This helps explain the remarkable photophysical properties of iron-carbenes in terms of unconventional electronic structure properties and favorable metal-ligand bonding interactions-important for the continued development of these type of complexes toward light-harvesting and light-emitting applications.

\section{INTRODUCTION}

Photofunctional transition metal complexes are used for a wide range of molecule-based light-harvesting and light-emitting applications that are currently under development. ${ }^{1}$ This includes solar energy conversion systems that cover both molecular photovoltaics and solar fuel applications, which take advantage of favorable and often unique excited-state dynamics and photo-induced charge-transfer properties of such complexes. ${ }^{2}$ Current efforts aim to replace rare and expensive metals in benchmark complexes (e.g., $\mathrm{Ru}^{2+}$ polypyridyl dyes ${ }^{3}$ ) with earth-abundant alternatives based on, for example, iron or copper. $^{4,5}$

Iron $\mathrm{N}$-heterocyclic carbene (NHC) complexes have recently emerged as a promising new class of photosensitizers ${ }^{6}$ capable of driving ultrafast electron injection on surfaces ${ }^{7}$ as well as bimolecular photoredox processes in solution. ${ }^{8}$ Specifically, the recently reported $\left[\mathrm{Fe}(\mathrm{btz})_{3}\right]^{3+}$ complex (Scheme 1), where btz is $3,3^{\prime}$-dimethyl-1,1' $1^{\prime}$-bis ( $p$-tolyl)-4, $4^{\prime}$ bis(1,2,3-triazol-5-ylidene), has a $\sim 100$ ps excited-state lifetime and is effectively the first reported photoluminescent ironbased light-harvesting and light-emitting complex. ${ }^{9}$ This constitutes a significant improvement over traditional iron complexes which suffer from ultrafast decay of initially excited charge-transfer states. ${ }^{10}$ This improvement is generally attributed to the electronic interaction between the iron center and the carbene ligands, where the ligands help to prolong the excited-state lifetime of the charge-transfer states.

The $\left[\mathrm{Fe}(\mathrm{btz})_{3}\right]^{3+}$ complex has been characterized as a low spin- $3 \mathrm{~d}^{5}$ complex, ${ }^{9}$ with the main metal and ligand frontier molecular orbital (MO) interactions schematically represented
Scheme 1. (a) Chemical Structure of $\mathrm{Fe}(\mathrm{btz})_{3} \cdot{ }^{14}$ (b) Geometry of a Single Ligand Showing the Bistriazole (btz) Core Carbene Moiety and Toluene Side-Groups
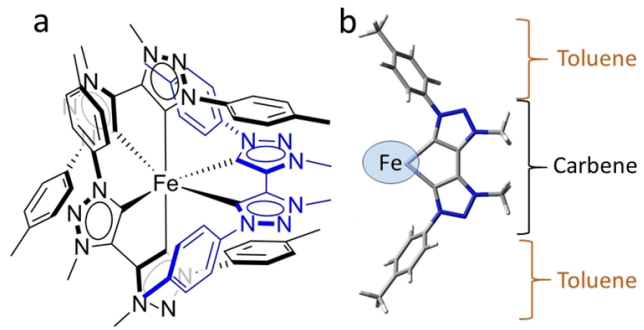

in Figure 1. An increased understanding of the influence of metal-ligand bonding and the charge-transfer properties is thus critical for further molecular design development. Particularly interesting for further investigations are the noninnocent contributions of the NHC-ligand $\sigma$ and $\pi$ orbitals with strong $\sigma$-donation and $\pi$-backbonding interactions with the metal center. This additional detail about the fundamental electronic properties enables comparisons with existing investigations of prototype iron-based photoactive complexes. ${ }^{11-13}$ There is therefore great interest in gaining

Received: January 29, 2020

Published: February 3, 2020 


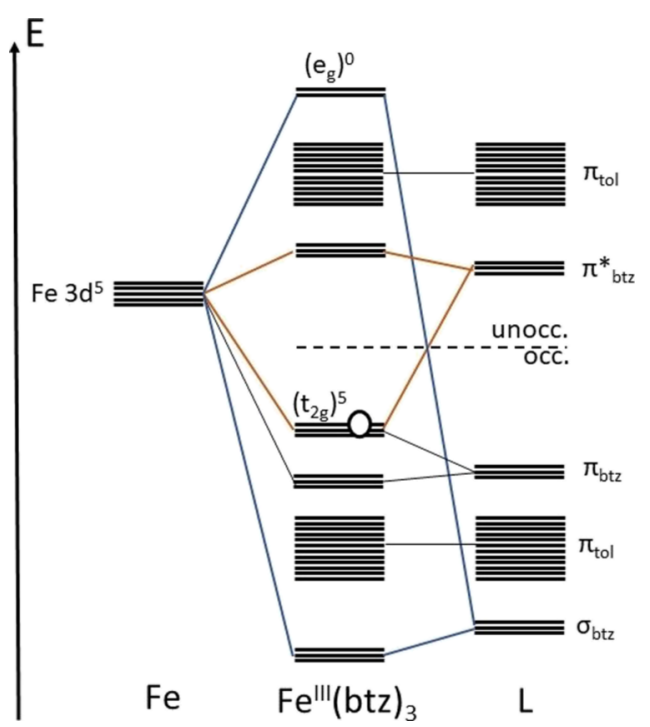

Figure 1. Schematic diagram of the molecular valence electronic structure of $\mathrm{Fe}^{\mathrm{III}}(\mathrm{btz})_{3}$ (in the quasi-octahedral ligand field-splitting convention) showing key orbital interactions between Fe 3d, carbenetype (btz) ligand (L), and toluene (tol) side-groups. Included are (i) the $\mathrm{Fe}^{\mathrm{III}} \mathrm{t}_{2 \mathrm{~g}}$ hole (empty circle), (ii) the carbene $\sigma$ lone pair bonding interaction with the unoccupied $\mathrm{Fe} \mathrm{e}_{\mathrm{g}}$ levels, and associated antibonding destabilization of the Fe $\mathrm{e}_{\mathrm{g}}$ level (blue lines), (iii) the Fe $t_{2 g}$ back-bonding interactions with carbene (btz) $\pi$ levels together with associated antibonding interactions (orange lines), and (iv) toluene (tol) side-group occupied $\pi$ and unoccupied $\pi^{*}$ orbitals.

better insight into the character and localizations of key MOs in this genre of $\mathrm{Fe}^{\mathrm{III}}$ complexes.

X-ray absorption spectroscopy (XAS) has proven to be a powerful tool to probe frontier orbital covalency, including spectral features resulting from complex multistate effects and ligand-metal interactions. ${ }^{12,15}$ Resonant X-ray photoelectron spectroscopy (RPES) provides complementary information, connecting unoccupied and occupied states, and has been used to study the electronic structure of components of solar energy devices, ${ }^{16-18}$ to deconstruct the valence band structure by the resonant enhancement of MOs, ${ }^{19-21}$ and even to measure ultrafast charge transfer. ${ }^{22,23}$ By using RPES alongside density functional theory (DFT) calculations, this paper explores the nature and localization of occupied and unoccupied MOs for an ultrathin film of $\left[\mathrm{Fe}(\mathrm{btz})_{3}\right]^{3+}$ through selective enhancement of orbitals localized to different regions in the molecule.

\section{EXPERIMENTAL METHODS}

Films of $\mathrm{Fe}(\mathrm{btz})_{3}\left(\mathrm{PF}_{6}\right)_{3}$ were dropcast onto clean gold substrates and were characterized by lab source X-ray photoelectron spectroscopy (XPS) (Physical Electronics PHI $\mathrm{X}$-Tool) and optical absorption spectroscopy (PerkinElmer Lambda 1050 spectrophotometer). RPES and near-edge X-ray absorption fine structure (NEXAFS) measurements were carried out at the HE-SGM beamline/endstation ${ }^{24}$ at the Helmholtz-Zentrum Berlin BESSY II synchrotron facility. Binding energy (BE) scales were calibrated to the gold fermi edge. Photon energy scales were calibrated using photoemission peaks generated from the first and second order light for the measurements over the $\mathrm{C}$ and $\mathrm{N}$ absorption edges. Further information regarding the instrumentation, sample preparation, and analysis methods can be found in the Supporting Information.

\section{RESULTS}

Figure 2 presents the optical absorbance spectra of a $\left[\mathrm{Fe}(\mathrm{btz})_{3}\right]^{3+}$ thin film on a gold substrate for comparison

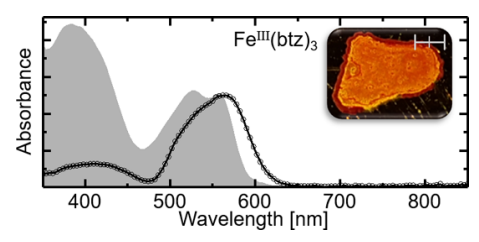

Figure 2. Absorbance of solution (colored area) and thin film (circles and lines) of $\left[\mathrm{Fe}(\mathrm{btz})_{3}\right]^{3+}$. Each curve is normalized to the peak at the longest wavelength. Inset: Photo of the as-prepared thin film with 1 $\mathrm{mm}$ scale bar.

with the previously characterized solutions of the complexes. ${ }^{9,14}$ The oxidation state of $\left[\mathrm{Fe}(\mathrm{btz})_{3}\right]^{3+}$ remains unchanged upon drop-casting. This is supported by XPS of the same films. Both measurements are further discussed in the Supporting Information. We are therefore proceeding with confidence that the $[\mathrm{Fe}(\mathrm{btz})]^{3+}$ films behave as previously characterized., ${ }^{9,14}$ This significant finding highlights the potential of $\left[\mathrm{Fe}(\mathrm{btz})_{3}\right]^{3+}$ for application in devices where solid thin films of the complex would be required.

As a basis for understanding the general electronic structure, an MO diagram of $\left[\mathrm{Fe}(\mathrm{btz})_{3}\right]^{3+}$ is presented in Figure 3 showing results from spin-polarized (unrestricted) DFT calculations performed on the B3LYP*/SDD/MeCN// B3LYP*/6-311G(d)/MeCN level of theory (detailed in the Supporting Information). These calculations provide a more complete MO perspective of the characteristic $3 \mathrm{~d}^{5}$ electronic configuration of the $\left[\mathrm{Fe}(\mathrm{btz})_{3}\right]^{3+}$ complex and the frontier $\mathrm{MO}$ structure that we have previously reported. ${ }^{9,25}$ Further details of the MO structure is presented in Supporting Information Figures S9-S12. The overall characteristics corroborate the previous findings in terms of the main metal and ligand $\mathrm{MO}$ features, including the low/spin $\left(t_{2 g}\right)^{5}$ population of the $\mathrm{Fe}$ (III) as well as significant metal-ligand mixing of several of the frontier orbitals, highlighting the noninnocent character introduced by the NHC-ligands for these complexes. The right-hand half of Figure 3 provides simple assignments of the experimental NEXAFS and RPES measurements with dominant $\mathrm{MO}$ contributions from the ground-state calculations (further supported by comparisons with information from explicit XAS calculations detailed in the Supporting Information), highlighting key resonant transitions from core levels to unoccupied states as discussed below.

$\mathrm{N}$ 1s and $\mathrm{C}$ 1s RPES maps presented in Figure 4 provide detailed site-specific insight into ligand MO features. Constant $\mathrm{BE}$ line profiles extracted from each map are included (showing NEXAFS constructed from photoelectrons originating in specific occupied orbitals). These are compared to conventional NEXAFS measured using the partial yield detector and simulated spectra (constructed from quantum chemical calculations-details can be found in the Supporting Information). The line shapes predicted by the calculations closely match the experimental spectra, providing support to the following assignments of spectral features.

$\mathrm{N}$ K-edge NEXAFS and RPES show two absorption resonances at 400.4 and $401.7 \mathrm{eV}$ photon energy. We assign these to absorption into the same unoccupied MO from two distinguishable core level environments labeled $N_{\mathrm{a}}$ and $N_{\mathrm{b}, \mathrm{c}}$ in 


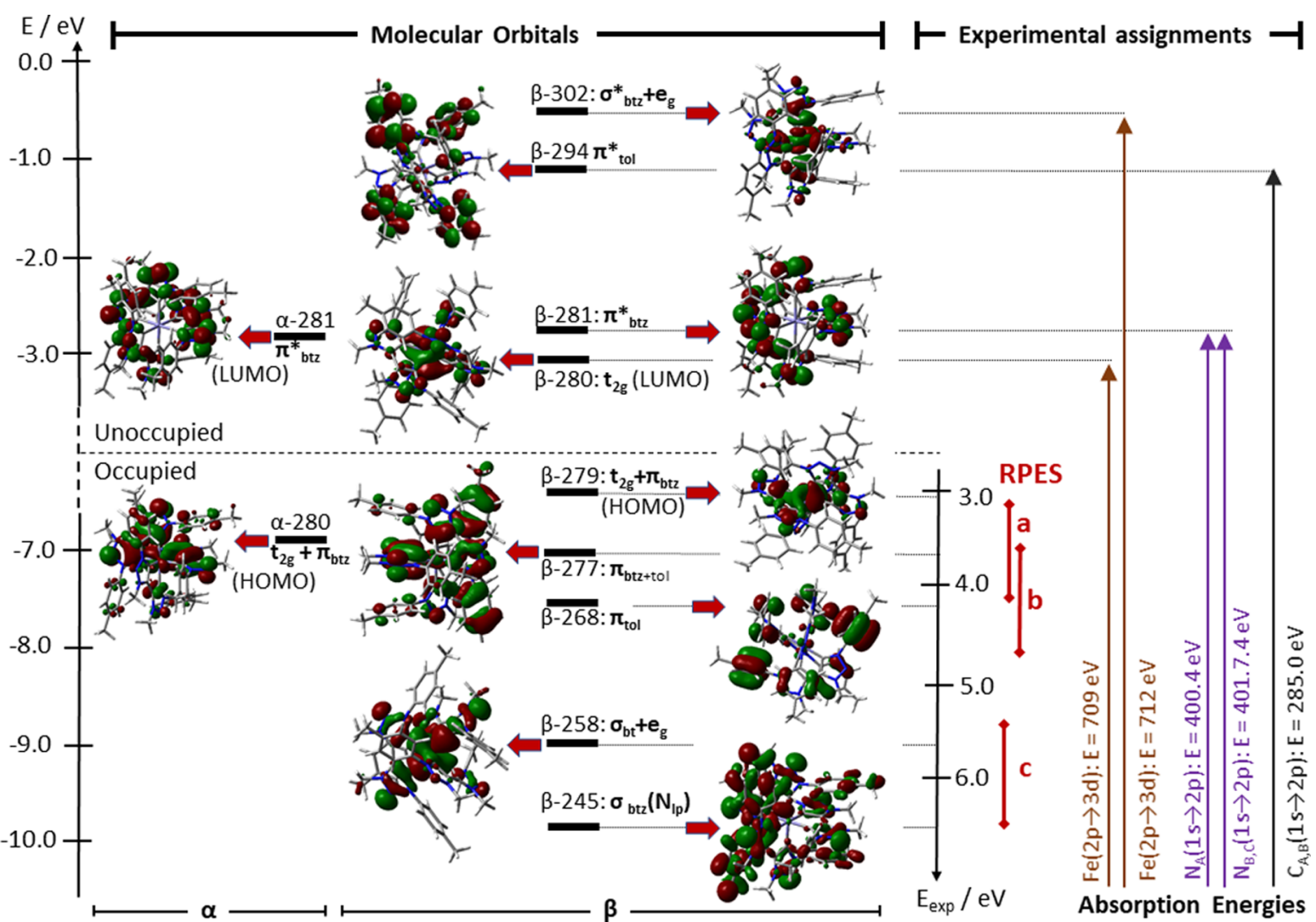

Figure 3. Selected valence MOs from spin-polarized electronic structure calculations of $\left[\mathrm{Fe}(\mathrm{btz})_{3}\right]^{3+}$ (left) together with associated assignments of experimental NEXAFS and RPES features to characteristic MO types (right). Alignment of experimental and calculated energy scales was done using the position of the $\pi_{\text {toluene }}$ orbital $(\beta-268)$.
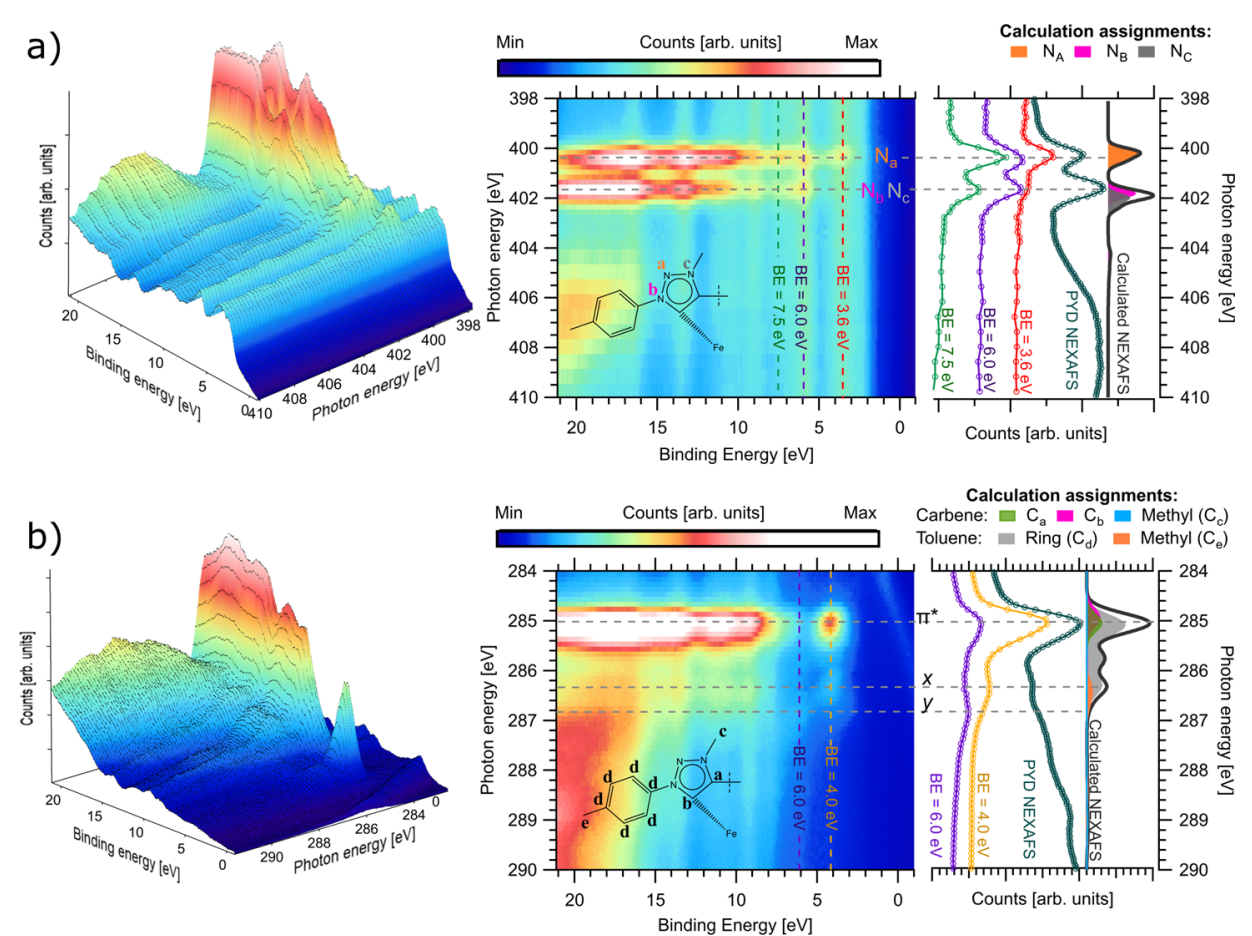

Figure 4. N 1s (a) and C 1s (b) RPES maps showing the valence band photoemission as a function of the incident photon energy. Alongside each are the constant $\mathrm{BE}$ line profiles extracted from the maps and NEXAFS, measured using a partial yield detector and simulated NEXAFS. The inset is the schematics of one of the six identical carbene rings and attached toluene groups with the different species labeled.

the inset in Figure 4a. This is consistent with both the simulated and measured NEXAFS which are in good agreement where the ratios of the two peaks in the spectra match the 1:2 ratio of the $N_{\mathrm{a}} / N_{\mathrm{b}, \mathrm{c}}$ atoms. It is also consistent 
with our interpretation of the XPS results (see Supporting Information). This unoccupied state is well described by the calculated MO $\beta$-281, which is evenly distributed around the nitrogen atoms (see Figure 3 ). The RPES line profiles show coupling of these NEXAFS features with the first three distinct occupied $\mathrm{MO}$ features at binding energies $3.6 \mathrm{eV}, 6.0 \mathrm{eV}$, and $7.5 \mathrm{eV}$, which are enhanced in the maps via participant decay. $^{26,27}$ The first $3.6 \mathrm{eV}$ feature shows strong enhancement/ coupling with the $N_{\mathrm{a}}$ atom and little coupling with the $N_{\mathrm{b}} / N_{\mathrm{c}}$ atoms. The feature at $6.0 \mathrm{eV} \mathrm{BE}$ is equally enhanced on excitation into either nitrogen environment. Finally, the $7.5 \mathrm{eV}$ enhancement shows stronger coupling with the $N_{\mathrm{a}}$ environment relative to the $N_{b, c}$ environment. Together, the results for the $\mathrm{N}$ 1s measurements highlight the atomic specificity of the probed valence MOs pertaining to their general molecular distribution and nodal structure, where there are clearly discernible differences for the different nitrogen environments on the btz rings.

The carbon NEXAFS (Figure 4b) represents a complex convolution of many different core levels and unoccupied orbitals, but the calculated spectra and RPES can aid in deconstructing it. The dominant feature is a strong absorption at $285.0 \mathrm{eV}$ corresponding to transitions from $\mathrm{C}$ on the toluene ligands into a set of $\pi^{*}$ MOs such as $\beta-294$ (almost exclusively localized on the toluene). The simulated spectra highlight that although this absorption feature is dominated by absorption into the toluene ring, the two carbons in the carbene ring also contribute. The absorption feature at $286.3 \mathrm{eV}$, labeled " $x$," appears to mainly originate from the toluene carbons (both the ring and methyl).

Constant BE line profiles have been extracted from the $\mathrm{C} 1 \mathrm{~s}$ map-this time intercepting the strong enhancement at $\mathrm{BE}=$ $4.2 \mathrm{eV}$, attributed to the $\pi$ orbital of the toluene, and the same $6 \mathrm{eV}$ feature highlighted in the $\mathrm{N}$ map. In the $\pi_{\text {tol }}(\mathrm{BE} \approx 4 \mathrm{eV})$ line profile, we see enhancement of the $\pi_{\mathrm{tol}}{ }^{*}$ and " $x$ " features. On the $6 \mathrm{eV}$ line, we see a new feature centered at $286.8 \mathrm{eV}$, labelled as " $y$ ". Unfortunately, we are unable to confidently identify the MO corresponding to this feature because the relative energy scales between experiment and theory drift apart as we stray further from the lowest unoccupied $\mathrm{MO}$ (LUMO).

Figure 5 presents resonantly enhanced valence band spectra, measured for $\mathrm{Fe}, \mathrm{N}$ and $\mathrm{C}$ resonances, on a common $\mathrm{BE}$ scale. This provides a basis for an in-depth analysis of occupied valence features and how these correlate between different elements/functional groups. Assignments of resonantly enhanced features require caution, for example, to distinguish between participant processes observed as constant BE features and resonant enhancement of Auger decay processes seen as constant kinetic energy features. Although it is common to interpret RPES in the simple MO perspective used here, it should also be noted that valence band features may also be affected by more complex multielectron state effects. ${ }^{28}$

The $\mathrm{C}$ and $\mathrm{N}$ valence band spectra in Figure 5 are extracted from the RPES maps at the photon energies previously highlighted to be of interest $\left(N_{\mathrm{a}} / N_{\mathrm{b}, \mathrm{c}}\right.$ and $\left.\pi_{\mathrm{tol}} * / x / y\right)$. The relevant sections of the maps are insets in the figure where these constant photon energy slices are marked. Fe maps are not presented because of severe radiation damage seen after extended resonant excitation of the Fe atom. Key excitation energies were therefore selected, and NEXAFS measurements were used to confirm that the radiation damage was minor. We also include valence band spectra measured off-resonance for

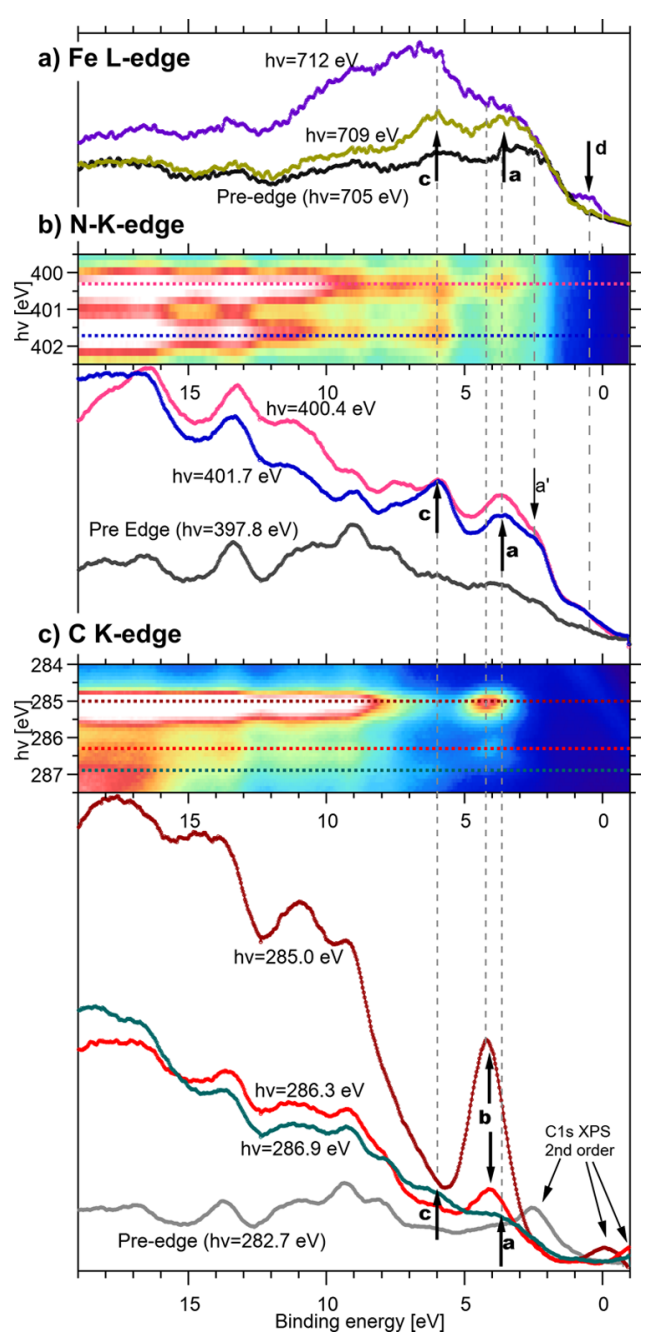

Figure 5. Valence band resonant enhancements on a shared BE scale. (a) 709 and $712 \mathrm{eV}$ represent $\mathrm{Fe}$ absorption into $t_{2 g}$ and $e_{g}$, respectively. (b,c) show the nitrogen and carbon RPES maps. Constant photon energy line profiles are extracted from (marked with dotted lines) and plotted under each map.

comparison-this aids in differentiating features enhanced by participant decay from the effect of variance in photoionization cross-sections with photon energy.

In the nitrogen and carbon data, three enhancements of occupied orbitals are highlighted (labeled a-c). Enhancements "a" and "c" are evident in the nitrogen RPES plots measured at photon energies of 400.4 and $401.7 \mathrm{eV}$. These enhancements are only weakly visible in the carbon RPES, and even then, only for the $286.9 \mathrm{eV}$ excitation data (corresponding to feature " $y$ " in the resonant NEXAFS-Figure 4b). Enhancement "a" $(\mathrm{BE} \approx 3.8 \mathrm{eV})$ is consistent with the calculated MO $\beta-277$, which has notable density on the iron atom, the innermost carbon, and the outermost nitrogen $\left(N_{\mathrm{a}}\right)$ atoms. Enhancement "c" $(\mathrm{BE} \approx 6.0 \mathrm{eV})$ is well represented by the $\beta-258 \mathrm{MO}$, which is localized across both $N_{\mathrm{a}}$ and $N_{\mathrm{b}, \mathcal{c}}$ the Fe center, and the carbene carbons. It follows that enhancement " $y$ " is a transition from carbon atoms in the carbene rings.

RPES enhancement " $\mathrm{b}$ " ( $\left.\mathrm{BE}=4.2 \mathrm{eV}, \pi_{\text {toluene }}\right)$, which is well represented by MO $\beta-286$, features strongly in the two line profiles at $h \nu=285 \mathrm{eV}\left(\pi^{*}\right)$ and $h \nu=286.3 \mathrm{eV}$ (“ $x$ ”). This supports that these two resonances correspond to LUMO levels localized on the toluene ligands. 
Excitation from $\mathrm{Fe} 2 \mathrm{p}$ to $3 \mathrm{~d}$ allows us to access both the $t_{2 \mathrm{~g}}$ (which has one electron vacancy) and the unoccupied $\mathrm{e}_{\mathrm{g}}$ levels. This is consistent with the Fe NEXAFS (Supporting Information S8) where we see a $3 \mathrm{eV}$ splitting between the spectral features attributed to the $t_{2 g}$ and $e_{g}$ levels at 709 and $712 \mathrm{eV}$, respectively. These features were investigated using RPES. When exciting into $\mathrm{e}_{\mathrm{g}}(h \nu=712 \mathrm{eV})$, we see a low BE feature, labeled $\mathrm{d}$, as well as enhancement of various unresolved higher energy valence features, which overlap with the Fe LMM auger decay (at $\sim 703 \mathrm{eV}$ kinetic energy ${ }^{29}$ ). When on-resonance with the $t_{2 g}$ hole $(h \nu=709 \mathrm{eV})$, we see enhancement in the same regions as the "a" (and lower energy shoulder labeled $a^{\prime}$ ) and "c" enhancements. This implies mixing/overlap between the $t_{2 g}$ and the highest occupied $\mathrm{MO}$ on the btz ligands, consistent with $\mathrm{MO} \beta$-279. Importantly, the covariation in resonance enhancement features between the $\mathrm{Fe}$ and btz in the low BE region (feature "a") suggests that the $t_{2 g}$ levels mix extensively with the $\pi_{\mathrm{btz}}$ orbitals. This is reminiscent of the well-known $\mathrm{Ru}-\mathrm{N} 3$ dye where there is significant orbital mixing between the $\mathrm{Ru} \mathrm{t}_{2 \mathrm{~g}}$ and $\pi$-levels on the NCS ligand. ${ }^{2}$

The low BE feature " $d$ " (visible when exciting into the $e_{g}$ ) remains intriguing as this enhancement is not apparent, when on the $t_{2 g}$ resonance or in the off-resonance spectra. It is also apparent in the $\mathrm{N}$ 1s RPES. The $\mathrm{C}$ 1s photoemission peaks generated from second-order light mean that we are unable to determine if it is apparent in the carbon RPES. RPES of highspin solid-state systems, such as hematite, ${ }^{30}$ have a feature attributed to the occupied $\mathrm{e}_{\mathrm{g}}$ at similar $\sim 1 \mathrm{eV} \mathrm{BE}$ we see in the RPES in this manuscript. For the low-spin system studied here, $\mathrm{e}_{\mathrm{g}}$ should be initially unoccupied and not produce photoemission features. This feature could be an intrinsic low BE feature of the $\mathrm{Fe}^{\mathrm{III}}$ species or could be due to the presence of a minority species (e.g., some X-ray-induced reduction to $\mathrm{Fe}^{\mathrm{II}}$ ). A more detailed investigation of this feature, including complete Fe RPES maps, would therefore be a valuable future study.

The overall experimental indications of strong metal-ligand orbital mixing for several of the frontier MO features involving the highest occupied and some of the low-lying unoccupied orbitals highlight the unique nature of the NHC-ligands in terms of $\sigma / \pi$ interactions with the $\mathrm{Fe} 3 \mathrm{~d}$ orbitals that has contributed to give these complexes remarkable photophysical properties. For example, the metal-ligand orbital mixing is important in terms of its influence on the energies of the $t_{2 g}$ levels beyond the generic ligand field-splitting model. The combination of the orbital specific and site-selective probing of key MO contributions, both occupied and unoccupied, also provides new insight into the unconventional electronic structure of this low-spin $3 \mathrm{~d}^{5}$ complex, where the lowest valence excitations are of ligand-to-metal charge-transfer character, rather than metal-to-ligand character that is common for many $\mathrm{Fe}(\mathrm{II})$ and $\mathrm{Ru}(\mathrm{II})$ photosensitizers with a $\mathrm{d}^{6}$ groundstate electronic configuration. In particular, the experimental determination of strong metal-ligand mixing is promising for further synthetic developments as it strongly suggests that key electronic properties, such as the $\mathrm{Fe}(\mathrm{II}) / \mathrm{Fe}$ (III) oxidation propensity and low-energy charge-transfer excitation properties, can be tuned via further ligand modifications.

\section{CONCLUSIONS}

We have made a critical first step toward extending the usefulness of iron-carbene photosensitizers from solutionbased applications to light-harvesting and light-emitting applications relying on thin-film technologies. Optical spectroscopy and XPS show that the oxidation state and the electronic configuration of the complex remain intact after drop-casting ultrathin films of the promising hexa-carbene photosensitizer $\mathrm{Fe}^{\mathrm{III}}(\mathrm{btz})_{3}$ onto a gold surface. RPES enabled us to probe, in several cases with atomic specificity, the coupling/localization of occupied and unoccupied MOs. These results are interpreted in the context of DFT calculations, providing detailed analysis of the frontier MOs involved in the excitation and emission processes. This reveals significant differences in the valence electronic interactions between the metal ion and the ligand carbene and auxiliary ligand orbitals. These results, in particular, provide direct spectroscopic evidence for strong MO interactions between the Fe 3d-levels and the btz carbene ligands' frontier $\sigma$ and $\pi$ orbitals. This is shown by resonant enhancement of the same MOs at the nitrogen, carbon, and iron absorption edges and by extensive mixing in the calculated MOs. Clear differences in the frontier orbital interactions between the iron and the different nitrogen atoms in the btz ring, revealed by the RPES measurements, highlight significant possibilities to alter the photophysics of the iron-carbene photosensitizers, as well as their interactions via linker and anchor groups, with atomic precision in a systematic and site-specific fashion. From a broader perspective, the improved understanding of the electronic structure properties of these materials thus paves the way for further development toward better photofunctional earthabundant materials based on iron and related transition metal complexes.

\section{ASSOCIATED CONTENT}

\section{Supporting Information}

The Supporting Information is available free of charge at https://pubs.acs.org/doi/10.1021/acs.jpca.0c00803.

Experimental methods, optical spectroscopy, oxidation state discussion, supporting XPS data, Fe L-edge data, radiation damage study, quantum chemical calculation methods and results, including density of states, decomposition of MOs, and table of results, detailed zoom of specific MOs, and explicit XAS calculations (PDF)

\section{AUTHOR INFORMATION}

\section{Corresponding Authors}

Jens Uhlig - Division of Chemical Physics, Chemical Center, Lund University, Lund SE-221 00, Sweden; 이잉.org/00000002-0528-0422; Email: Jens.Uhlig@chemphys.lu.se

Joachim Schnadt - Division of Synchrotron Radiation Research, Department of Physics, MAX IV Laboratory, Lund University, Lund SE-22100, Sweden; 이이.org/0000-0001-9375831X; Email: joachim.schnadt@sljus.lu.se

Petter Persson - Theoretical Chemistry Division, Chemical Center, Lund University, Lund SE-221 00, Sweden; ○ orcid.org/0000-0001-7600-3230; Email: Petter.Persson@ teokem.lu.se

\section{Authors}

Robert H. Temperton - School of Physics and Astronomy, University of Nottingham, Nottingham NG7 2RD, U.K.; (1) orcid.org/0000-0002-4802-6862

Nils W. Rosemann - Division of Chemical Physics, Chemical Center and Centre for Analysis and Synthesis, Department of 
Chemistry, Lund University, Lund SE-221 00, Sweden; (1) orcid.org/0000-0002-7663-0397

Meiyuan Guo - Division of Chemical Physics, Chemical Center, Lund University, Lund SE-221 00, Sweden; 이이이.org/00000003-2474-6264

Niclas Johansson - Division of Synchrotron Radiation Research, Department of Physics, MAX IV Laboratory, Lund University, Lund SE-22100, Sweden

Lisa A. Fredin - Theoretical Chemistry Division, Chemical Center, Lund University, Lund SE-221 00, Sweden; (1) orcid.org/0000-0002-4091-0899

Om Prakash - Centre for Analysis and Synthesis, Department of Chemistry, Lund University, Lund SE-22100, Sweden; (ㄷ) orcid.org/0000-0002-2322-6403

Kenneth Wärnmark - Centre for Analysis and Synthesis, Department of Chemistry, Lund University, Lund SE-22100,

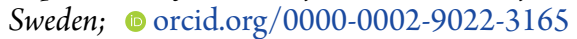

Karsten Handrup - Division of Synchrotron Radiation Research, Department of Physics, MAX IV Laboratory, Lund University, Lund SE-22100, Sweden

Complete contact information is available at: https://pubs.acs.org/10.1021/acs.jpca.0c00803

\section{Notes}

The authors declare no competing financial interest.

\section{ACKNOWLEDGMENTS}

We acknowledge the Helmholtz-Zentrum-Berlin (HZB) and the staff at BESSY for generously supporting our measurements. R.H.T. acknowledges the financial support from the Engineering and Physical Sciences Research Council (EPSRC) and the University of Nottingham Propulsion Futures Beacon. P.P. acknowledges the financial support from the Swedish Research Council (VR), the Swedish Energy Agency (Energimyndigheten), the Swedish Foundation for Strategic Research (SSF), the Knut and Alice Wallenberg Foundation (KAW), NanoLund, and eSSENCE as well as the LUNARC and NSC national supercomputer centers for providing computational resources via SNIC. J.U. gratefully acknowledges the financial support from the Carl Tryggers stiftelse and Crafoord Stiftelse. N.W.R. gratefully acknowledges funding from the Alexander von Humboldt Foundation within the Feodor-Lynen Fellowship program. We thank Prof. Per Persson and Michiel Op de Beeck of the Biology Department, Lund University, for access to XPS measurement facilities.

\section{REFERENCES}

(1) Balzani, V.; Bergamini, G.; Campagna, S.; Puntoriero, F. Photochemistry and Photophysics of Coordination Compounds: Overview and General Concepts. In Photochemistry and Photophysics of Coordination Compounds I; Balzani, V., Campagna, S., Eds.; Springer Berlin Heidelberg: Berlin, Heidelberg, 2007; pp 1-36.

(2) Ponseca, C. S., Jr.; Chábera, P.; Uhlig, J.; Persson, P.; Sundström, V. Ultrafast Electron Dynamics in Solar Energy Conversion. Chem. Rev. 2017, 117, 10940-11024.

(3) Grätzel, M. Dye-Sensitized Solar Cells. J. Photochem. Photobiol., C 2003, 4, 145-153.

(4) Bozic-Weber, B.; Constable, E. C.; Housecroft, C. E. Light Harvesting with Earth Abundant D-Block Metals: Development of Sensitizers in Dye-Sensitized Solar Cells (DSCs). Coord. Chem. Rev. 2013, 257, 3089-3106.

(5) Wenger, O. S. Photoactive Complexes with Earth-Abundant Metals. J. Am. Chem. Soc. 2018, 140, 13522.
(6) Liu, Y.; Persson, P.; Sundström, V.; Wärnmark, K. Fe NHeterocyclic Carbene Complexes as Promising Photosensitizers. Acc. Chem. Res. 2016, 49, 1477-1485.

(7) Harlang, T. C. B.; Liu, Y.; Gordivska, O.; Fredin, L. A.; Ponseca, C. S., Jr; Huang, P.; Chábera, P.; Kjaer, K. S.; Mateos, H.; Uhlig, J.; et al. Iron Sensitizer Converts Light to Electrons with $92 \%$ Yield. Nat. Chem. 2015, 7, 883-889.

(8) Kjær, K. S.; Kaul, N.; Prakash, O.; Chábera, P.; Rosemann, N. W.; Honarfar, A.; Gordivska, O.; Fredin, L. A.; Bergquist, K.-E.; Häggström, L.; et al. Luminescence and Reactivity of a ChargeTransfer Excited Iron Complex with Nanosecond Lifetime. Science 2019, 363, 249.

(9) Chábera, P.; Liu, Y.; Prakash, O.; Thyrhaug, E.; Nahhas, A. E.; Honarfar, A.; Essén, S.; Fredin, L. A.; Harlang, T. C. B.; Kjær, K. S.; et al. A Low-Spin Fe(III) Complex with 100-Ps Ligand-to-Metal Charge Transfer Photoluminescence. Nature 2017, 543, 695-699.

(10) Monat, J. E.; McCusker, J. K. Femtosecond Excited-State Dynamics of an Iron(II) Polypyridyl Solar Cell Sensitizer Model. J. Am. Chem. Soc. 2000, 122, 4092-4097.

(11) Zhang, W.; Gaffney, K. J. Mechanistic Studies of Photoinduced Spin Crossover and Electron Transfer in Inorganic Complexes. Acc. Chem. Res. 2015, 48, 1140-1148.

(12) Baker, M. L.; Mara, M. W.; Yan, J. J.; Hodgson, K. O.; Hedman, B.; Solomon, E. I. K- and L.-Edge X-Ray Absorption Spectroscopy (XAS) and Resonant Inelastic X-Ray Scattering (RIXS) Determination of Differential Orbital Covalency (DOC) of Transition Metal Sites. Coord. Chem. Rev. 2017, 345, 182-208.

(13) Leshchev, D.; Harlang, T. C. B.; Fredin, L. A.; Khakhulin, D.; Liu, Y.; Biasin, E.; Laursen, M. G.; Newby, G. E.; Haldrup, K.; Nielsen, M. M.; et al. Tracking the Picosecond Deactivation Dynamics of a Photoexcited Iron Carbene Complex by TimeResolved X-Ray Scattering. Chem. Sci. 2018, 9, 405-414.

(14) Chábera, P.; Kjaer, K. S.; Prakash, O.; Honarfar, A.; Liu, Y.; Fredin, L. A.; Harlang, T. C. B.; Lidin, S.; Uhlig, J.; Sundström, V.; et al. Feii Hexa N-Heterocyclic Carbene Complex with a 528 Ps Metal-to-Ligand Charge-Transfer Excited-State Lifetime. J. Phys. Chem. Lett. 2018, 9, 459-463.

(15) Wasinger, E. C.; de Groot, F. M. F.; Hedman, B.; Hodgson, K. O.; Solomon, E. I. L-Edge X-Ray Absorption Spectroscopy of NonHeme Iron Sites: Experimental Determination of Differential Orbital Covalency. J. Am. Chem. Soc. 2003, 125, 12894-12906.

(16) Rensmo, H.; Södergren, S.; Patthey, L.; Westermark, K.; Vayssieres, L.; Kohle, O.; Brühwiler, P. A.; Hagfeldt, A.; Siegbahn, H. The Electronic Structure of the Cis-bis $\left(4,4^{\prime}\right.$-Dicarboxy-2,2'-Bipyridine)-bis(isothiocyanato)ruthenium(II) Complex and Its Ligand 2,2'-Bipyridyl-4,4'-Dicarboxylic Acid Studied with Electron Spectroscopy. Chem. Phys. Lett. 1997, 274, 51-57.

(17) Persson, P.; Lunell, S.; Brühwiler, P. A.; Schnadt, J.; Södergren, S.; O’Shea, J. N.; Karis, O.; Siegbahn, H.; Mårtensson, N.; Bässler, M.; et al. N 1s X-Ray Absorption Study of the Bonding Interaction of BiIsonicotinic Acid Adsorbed on Rutile TiO2(110). J. Chem. Phys. 2000, 112, 3945-3948.

(18) Cappel, U. B.; Lanzilotto, V.; Johansson, E. M. J.; Edvinsson, T.; Rensmo, H. X-Ray Photoelectron Spectroscopy for Understanding Molecular and Hybrid Solar Cells. In Molecular Devices for Solar Energy Conversion and Storage; Tian, H., Boschloo, G., Hagfeldt, A., Eds.; Springer Singapore: Singapore, 2018; pp 433-476.

(19) Solomon, E. I.; Basumallick, L.; Chen, P.; Kennepohl, P. Variable Energy Photoelectron Spectroscopy: Electronic Structure and Electronic Relaxation. Coord. Chem. Rev. 2005, 249, 229-253.

(20) Green, J. C. Variable Photon Energy Photoelectron Spectroscopy of Transition Metal Molecules. Acc. Chem. Res. 1994, 27, 131137.

(21) Li, X.; Bancroft, G. M.; Puddephatt, R. J. Variable Energy Photoelectron Spectroscopy: Periodic Trends in D-Orbital Energies for Organometallic Compounds of the Transition Metals. Acc. Chem. Res. 1997, 30, 213-218.

(22) Schnadt, J.; Brühwiler, P. A.; Patthey, L.; O'Shea, J. N.; Södergren, S.; Odelius, M.; Ahuja, R.; Karis, O.; Bässler, M.; Persson, 
P.; et al. Experimental Evidence for Sub-3-Fs Charge Transfer from an Aromatic Adsorbate to a Semiconductor. Nature 2002, 418, 620-623.

(23) Gibson, A. J.; Temperton, R. H.; Handrup, K.; Weston, M.; Mayor, L. C.; O’Shea, J. N. Charge Transfer from an Adsorbed Ruthenium-Based Photosensitizer through an Ultra-Thin Aluminium Oxide Layer and into a Metallic Substrate. J. Chem. Phys. 2014, 140, 234708.

(24) Nefedov, A.; Wöll, C. Advanced Applications of NEXAFS Spectroscopy for Functionalized Surfaces. In Surface Science Techniques; Bracco, G., Holst, B., Eds.; Springer Berlin Heidelberg: Berlin, Heidelberg, 2013; pp 277-303.

(25) Ericson, F.; Honarfar, A.; Prakash, O.; Tatsuno, H.; Fredin, L. A.; Handrup, K.; Chabera, P.; Gordivska, O.; Kjær, K. S.; Liu, Y.; et al. Electronic Structure and Excited State Properties of Iron Carbene Photosensitizers - A Combined X-Ray Absorption and Quantum Chemical Investigation. Chem. Phys. Lett. 2017, 683, 559-566.

(26) Brühwiler, P. A.; Karis, O.; Mårtensson, N. Charge-Transfer Dynamics Studied Using Resonant Core Spectroscopies. Rev. Mod. Phys. 2002, 74, 703-740.

(27) Temperton, R. H.; Gibson, A. J.; Handrup, K.; O'Shea, J. N. Adsorption and Charge Transfer Interactions of Bi-Isonicotinic Acid on $\operatorname{Ag}(111)$. J. Chem. Phys. 2017, 147, 054703.

(28) Armen, G. B.; Aksela, H.; Åberg, T.; Aksela, S. The Resonant Auger Effect. J. Phys. B: At., Mol. Opt. Phys. 2000, 33, R49.

(29) Powell, C. J. Recommended Auger-Electron Kinetic Energies For 42 Elemental Solids. J. Electron Spectrosc. Relat. Phenom. 2010, 182, 11-18.

(30) Bora, D. K.; Hu, Y.; Thiess, S.; Erat, S.; Feng, X.; Mukherjee, S.; Fortunato, G.; Gaillard, N.; Toth, R.; Gajda-Schrantz, K.; et al. Between Photocatalysis and Photosynthesis: Synchrotron Spectroscopy Methods on Molecules and Materials for Solar Hydrogen Generation. J. Electron Spectrosc. Relat. Phenom. 2013, 190, 93-105. 